\title{
Molecular-genetic and Chemotaxonomic Studies on Actinomadura and Nocardiopsis
}

\author{
By ANGELIKA FISCHER, ${ }^{1}$ REINER M. KROPPENSTEDT ${ }^{2}$ AND \\ ERKO STACKEBRANDT ${ }^{1}$ * \\ ${ }^{1}$ Lehrstuhl für Mikrobiologie, Technische Universität München, Arcisstrasse 21, \\ D-8000 München 2, Federal Republic of Germany \\ ${ }^{2}$ Deutsche Sammlung von Mikroorganismen, Gesellschaft für Biotechnologische Forschung $\mathbf{m b H}$ \\ Braunschweig, Schnittspahnstrasse 9, D-6100 Darmstadt, Federal Republic of Germany
}

(Received 6 April 1983; revised 21 June 1983)

\begin{abstract}
The relationships of 24 strains of 13 species of Actinomadura and 4 strains of Nocardiopsis dassonvillei were determined by nucleic acid hybridization studies. DNA-rRNA cistron similarity and DNA homology values reveal that Actinomadura is genetically heterogeneous. One cluster contained the type species Actinomadura madurae, Actinomadura pelletieri, Actinomadura verrucosospora, Actinomadura malachitica, Actinomadura citrea and 'Actinomadura kijaniata'. A second cluster embraced Actinomadura pusilla, Actinomadura roseoviolacea, Actinomadura libanotica, Actinomadura roseola and Actinomadura ferruginea. The internal homogeneity of the two Actinomadura clusters was demonstrated by a high similarity in the menaquinone and fatty acid composition of the strains enclosed. Actinomadura spadix, Actinomadura spiralis, and two strains of Actinomadura madurae were found to be unrelated to each other and could not be allocated to one of the two major Actinomadura clusters. Nocardiopsis dassonvillei was genetically and phenotypically clearly separated from all Actinomadura species investigated.
\end{abstract}

\section{INTRODUCTION}

Some years ago we began to explore relatedness among various sporoactinomycete genera. The determination of DNA-DNA homologies allows the detection of relationships at the strain and species level, and DNA-rRNA cistron similarities determined by hybridization are valuable at the intrafamily level. Partial sequence analysis of $16 \mathrm{~S}$ rRNA can be used to define orders or even lower ranks. As a result of our studies a hierarchic structure of the sporoactinomycetes emerged (Stackebrandt \& Woese, $1981 a, b$; Stackebrandt et al., 1981, 1983; Stackebrandt \& Schleifer, 1983).

In most cases organisms in the clusters so obtained had a high similarity in characters of chemotaxonomic value, as seen with various streptomycete genera, the StreptosporangiumPlanomonospora-Planobispora group and the Actinoplanes group of organisms (Stackebrandt et al., 1981, 1983).

The genus Actinomadura is one of the sporoactinomycete genera rarely included in extensive chemotaxonomic and genetic investigations. This genus contains actinomycetes forming a stable mycelium, with or without an aerial mycelium bearing arthrospores of different morphology and having cell walls with meso-diaminopimelic acid (Lechevalier \& Lechevalier, 1970; Williams \& Wellington, 1981); most of the species are reported to contain madurose, identified as 3-O-methylgalactose (Lechevalier \& Gerber, 1970). Contradictory results have been reported on the DNA G $+\mathrm{C}$ content of the type species Actinomadura madurae, ranging from $65 \mathrm{~mol} \%$ (Mordarski et al., 1977) to $74.5 \mathrm{~mol} \%$ (Lechevalier et al., 1971). In the few comparative chemotaxonomic investigations which have been done on Actinomadura, in most 
cases only $A$. madurae and $A$. pelletieri, or only a small number of the more than 20 validly described species (Skerman et al., 1980; Horan \& Brodsky, 1982) were included in studies on isoprenoid quinones (Yamada et al., 1977, 1982; Collins et al., 1977; Collins \& Jones, 1981 ; Aoki et al., 1980), polar lipids (Minnikin et al., 1977), and fatty acids (Agre et al., 1975). Little information is therefore available about the homogeneity of Actinomadura. The first indication that this genus actually contains a heterogeneous collection of species came from the analysis of polar lipids (Agre et al., 1975) and from numerical phenetic studies (Goodfellow \& Pirouz, 1982).

In this communication we present data on a variety of chemotaxonomic characters, e.g. menaquinone composition, whole cell sugars, fatty acids of diagnostic value, and the DNA $\mathrm{G}+\mathrm{C}$ content of 13 species of Actinomadura and of Nocardiopsis dassonvillei, which has recently been excluded from Actinomadura on phenotypic grounds (Meyer, 1976).

\section{METHODS}

Bacteria used and culture conditions. The strains investigated, their sources and their DNA G + C content are listed in Table 1. For the isolation of high-molecular weight DNA, organisms were cultivated in broth containing $\left(\%\right.$, w/v) : peptone (Oxoid), 0.5 ; yeast extract (Oxoid), $0 \cdot 2$; sucrose, $3 \cdot 0 ; \mathrm{KNO}_{3}, 0 \cdot 2 ; \mathrm{K}_{2} \mathrm{HPO}_{4}, 0 \cdot 1 ; \mathrm{MgSO}_{4} .7 \mathrm{H}_{2} \mathrm{O}$, $0.05 ; \mathrm{KCl}, 0.05 ; \mathrm{pH} \mathrm{7.3}$. For the isolation of in vivo-labelled DNA and ribosomal RNA, cells were grown in the same broth, but the organic compounds were reduced to half the amounts listed above. All organisms were cultivated aerobically by shaking for 2 to $4 \mathrm{~d}$ at $28^{\circ} \mathrm{C}$. Bacteria were harvested by filtering the suspension through a Whatman no. 1 filter paper.

Isolation of nucleic acids. Unlabelled and labelled DNAs were purified according to Marmur (1961), modified as described by Meyer \& Schleifer (1978) and Stackebrandt et al. (1981). Ribosomal RNA (23S) was isolated as described by Stackebrandt $e t$ al. (1981). In vivo labelling of DNA and rRNA with $\left[{ }^{3} \mathrm{H}\right]$ adenine (New England Nuclear, $14.1 \mathrm{Ci} \mathrm{mmol}^{-1}, 522 \mathrm{GBq} \mathrm{mmol}{ }^{-1}$ ), using $0.5 \mathrm{mCi}$ and $1 \mathrm{mCi}$, respectively, was done as described by Stackebrandt et al. (1981). In vitro labelling of DNA by nick-translation with $50 \mu \mathrm{Ci}\left[{ }^{32} \mathrm{~S}\right] \mathrm{dATP}$ (New England Nuclear, $494 \mathrm{Ci} \mathrm{mmol}^{-1}, 18300 \mathrm{GBq} \mathrm{mmol}^{-1}$ ) was done using the nick-translation kit of Bethesda Research Laboratories (Cambridge, U.K.). The specific activities for DNA ranged between $8 \times 10^{3}$ and $2.5 \times$ $10^{4}$ c.p.m. $\left(\mu \mathrm{g}\left[{ }^{3} \mathrm{H}\right] \mathrm{DNA}\right)^{-1}$, and $1 \times 10^{5}$ and $7 \times 10^{5}$ c.p.m. $\left(\mu \mathrm{g}\left[{ }^{35} \mathrm{~S}\right] \mathrm{DNA}\right)^{-1}$, respectively; that for $23 \mathrm{~S} \mathrm{rRNA}$ was $6 \times 10^{4}$ c.p.m. $\left(\mu \mathrm{g}\left[{ }^{3} \mathrm{H}\right] \mathrm{RNA}\right)^{-1}$.

Hybridization procedures. DNA-DNA hybridization experiments were carried out in two different ways. The DNA-filter method was done as originally described by McConaughy et al. (1969) and Denhardt (1966), modified as described by Stackebrandt \& Kandler (1979). A sample $(0 \cdot 1 \mu \mathrm{g})$ of fragmented and denatured [ $\left.{ }^{3} \mathrm{H}\right] \mathrm{DNA}$ or ${ }^{[35}$ S]DNA in $0.2 \mathrm{ml} 3 \times \mathrm{SSC}(1 \times \mathrm{SSC}$ is $0.15 \mathrm{M}$-sodium chloride, $0.015 \mathrm{M}$-trisodium citrate, $\mathrm{pH} 7.0)$, adjusted to $33 \%$ formamide, was hybridized against 7 to $10 \mu \mathrm{g}$ filter-bound DNA for $20 \mathrm{~h}$ at $60^{\circ} \mathrm{C}$, corresponding to slightly 'stringent' reassociation conditions $\left(20^{\circ} \mathrm{C}\right.$ below $T_{m}$ of the DNA). When $\left[{ }^{35} \mathrm{~S}\right] \mathrm{DNA}$ was used as a reference DNA, the hybridization buffer contained $10 \mathrm{mM}$-dithiothreitol.

Alternatively, in some cases the DNA homology was determined spectrophotometrically by measuring the reassociation kinetics of DNA solutions (De Ley et al., 1970) in a recording spectrophotometer (Gilford, Model 2600). Purified DNA, dialysed twice against $2 \times$ SSC was fragmented by passing three times through a French pressure cell at $1.7 \mathrm{GPa}$ and adjusted to $50 \mu \mathrm{g} \mathrm{DNA} \mathrm{ml}^{-1}$. The DNA of Actinomadura and Nocardiopsis strains have an average $\mathrm{G}+\mathrm{C}$ content of $68 \mathrm{~mol} \%$, from which it follows that the melting point of the DNA in $2 \times \mathrm{SSC}$ is $101.5^{\circ} \mathrm{C}$ (Frank-Kamenetskii, 1971). The melting point of the DNA was decreased to $92{ }^{\circ} \mathrm{C}$ by the addition of $14 \%$ formamide to the DNA solution. Three DNA solutions (equal concentrations of DNAs of strain $A$ and of strain $B$ and a mixture of equal volumes of both DNA samples) and a buffer blank ( $2 \times$ SSC adjusted to $14 \%$ formamide) were heated at $97{ }^{\circ} \mathrm{C}$ for $10 \mathrm{~min}$ and adjusted to a temperature of $82^{\circ} \mathrm{C}$ within $2-3 \mathrm{~min}$. The hybridization temperature was calculated according to Gillis et al. (1970). Owing to the presence of $14 \%$ formamide in the reassociation solution, hybridization was carried out under stringent conditions $\left(10^{\circ} \mathrm{C}\right.$ below $T_{m}$ of the DNA).

DNA-23S rRNA hybridization and the determination of the melting points of the DNA-rRNA duplexes followed the method of De Ley \& De Smedt (1975), with the slight modifications described by Döpfer et al. (1982).

Determination of the $G+C$ mol $\%$ of the DNA. The guanine (G) plus cytosine (C) content of the DNA was calculated from its thermal denaturation temperature (Mandel \& Marmur, 1968). The melting profiles were determined in a Gilford 2600 spectrophotometer.

Cell wall analysis. Amino acids and sugars of cell material were released by acid hydrolysis (Becker et al., 1965; Lechevalier $e t$ al., 1971). The whole cell hydrolysates were separated by cellulose TLC with methanol/water/10 M$\mathrm{HCl} /$ pyridine ( $80: 17 \cdot 5: 2 \cdot 5: 10$, by vol.) as developing solvent in the case of the amino acids and with ethyl acetate/pyridine/water $(100: 35: 25$, by vol.) in the case of the sugars. The separated amino acids and sugars were identified with specific spraying reagents (Stanek \& Roberts, 1974). 
Extraction and analysis of fatty acid methyl esters. Freeze-dried cells were subjected to acid methanolysation (Minnikin et al., 1975). The fatty acid methyl esters were extracted with petrol ether, and purified by TLC on silica gel $\mathrm{PF}_{254+366}$ of $0.4 \mathrm{~mm}$ thickness. The developing solvent was petroleum ether (b.p. $60-80^{\circ} \mathrm{C}$ )/diethylether $(85: 15, \mathrm{v} / \mathrm{v})$. Under $\mathrm{UV}_{366^{-}}$-irradiation the fatty acid methyl esters are visible as light blue spots. The fatty acid methyl esters were eluted with chloroform and analysed with a Varian 2700 and a Hewlett Packard 5700 gas chromatograph. Both chromatographs were fitted with a splitter and auxiliary gas supply to run capillary columns. The conditions are the following: Varian fused silica capillary column $25 \mathrm{~m} \times 0.32 \mathrm{~mm}$ (WCOT), OV101, oven temperature $180^{\circ} \mathrm{C}$, inlet pressure $0.3 \mathrm{bar}(30 \mathrm{kPa})$, split $1: 40$; Hewlett Packard, fused silica capillary column $25 \mathrm{~m} \times 0.32 \mathrm{~mm}$ (WCOT), FFAP, oven temperature $180^{\circ} \mathrm{C}$, inlet pressure $1 \mathrm{bar}$, split $1: 20$, detector FID. The fatty acid methyl esters were identified by comparing their retention times with that of standard mixtures (Kroppenstedt \& Kutzner, 1978; Kroppenstedt, 1982a).

Extraction, purification and identification of isoprenoid quinones. Samples (100-200 mg) of freeze-dried cells were suspended in $2 \mathrm{ml}$ acetone, cooled in an ice bath, disrupted by ultrasonication for $2 \mathrm{~min}$ and separated by centrifugation. This procedure was repeated three times, and the acetone extracts were dried on a rotary evaporator at $40^{\circ} \mathrm{C}$ (Kroppenstedt et al., 1981). After the isolation of menaquinones with adsorption TLC on silica gel (Dunphy et al., 1971), the menaquinones were separated by HPLC on two different stationary phases (RP 18 and silver-loaded ion exchanger) and identified on their retention behaviour according to their isoprenylic chainlength and their degree of saturation (Kroppenstedt, 1982 b).

\section{RESULTS}

The $\mathrm{G}+\mathrm{C}$ mol\% of the DNA of 24 strains of 13 species of Actinomadura and 4 strains of Nocardiopsis dassonvillei ranged between 65.5 and $69.7 \%$ and 64 and $69 \%$, respectively (Table 1).

\section{DNA-rRNA hybridization}

Information about the phylogenetic coherence of the genus Actinomadura and about the relationship of this genus to Nocardiopsis, Streptomyces and Streptosporangium was achieved by the determination of the melting point of DNA-rRNA duplexes [ $T_{m(e)}$ values], obtained with ${ }^{3} \mathrm{H}$-labelled 23S rRNA of Actinomadura madurae DSM 43067 and DNA from representatives of the various genera (Table 2). In respect of the thermal stability of DNA-rRNA duplexes, strains of Actinomadura form two clusters. Organisms of the first cluster (here designated as A. maduraecluster) show high $T_{m(e)}$ values (79.0 to $84^{\circ} \mathrm{C}$ ) with reference rRNA of $A$. madurae DSM 43067, i.e. three strains of $A$. madurae, all strains of $A$. pelletieri investigated, $A$. verrucosospora DSM 43358, $A$. malachitica DSM 43462, $A$. citrea DSM 43661 and ' $A$. kijaniata' ATCC 31588. The second cluster embraces all other strains of Actinomadura investigated, including two strains classified as $A$. madurae. The thermal stability of the duplexes formed with DNA of strains of this second cluster and rRNA of $A$. madurae DSM 43067 is markedly lower (73.1$75.8^{\circ} \mathrm{C}$ ) than that found for members of the $A$. madurae-cluster. The $T_{m(e)}$ values of members of this second Actinomadura rRNA-cluster are in the same range as those found for duplexes formed by $A$. madurae DSM 43067 and four strains of Nocardiopsis dassonvillei $\left(72-73.7^{\circ} \mathrm{C}\right)$, Streptosporangium roseum DSM 43021, and Streptomyces griseus DSM 43236 (73.8 and 73.2, respectively).

\section{DNA-DNA hybridization}

Since the determination of relationships by rRNA cistron similarity experiments does not allow the detection of relationships between closely related species, organisms of the two Actinomadura rRNA-clusters were subjected to DNA-DNA hybridization experiments. The average binding efficiency in the homologous system was $28 \%$. DNA, labelled in vivo with $\left[{ }^{3} \mathrm{H}\right]$ adenine or in vitro with $\left[{ }^{35} \mathrm{~S}\right] \mathrm{dATP}$ by nick-translation showed the same binding efficiency. Homology values obtained by the spectrophotometrical method showed a high correspondence to those values obtained by the filter-method. The homology values are listed in Tables 3-6. Organisms of the $A$. madurae-rRNA cluster share homology values ranging from 18 to $100 \%$ (Tables 3 and 6). The highest values are found between $A$. madurae strains DSM 43067 and DSM $43380(96 \%)$, between various strains of $A$. pelletieri $(85-100 \%$ ) (Table 3 ), and between the two strains of $A$. verrucosospora (96\%) (Table 6). Except for $A$. madurae DSM 43122 and $A$. 
Table 1. Source and DNA $G+C$ content of the organisms investigated

Strain

Actinomadura madurae Actinomadura madurae Actinomadura madurae Actinomadura madurae Actinomadura madurae Actinomadura madurae Actinomadura pelletieri Actinomadura pelletieri Actinomadura pelletieri Actinomadura pelletieri Actinomadura pelletieri Actinomadura pusilla Actinomadura roseoviolacea Actinomadura verrucosospora Actinomadura verrucosospora Actinomadura spadix Actinomadura citrea Actinomadura malachitica Actinomadura roseola Actinomadura roseola Actinomadura ferruginea Actinomadura libanotica Actinomadura spiralis 'Actinomadura kijaniata' Nocardiopsis dassonvillei Nocardiopsis dassonvillei Nocardiopsis dassonvillei Nocardiopsis dassonvillei Streptosporangium roseum Streptomyces griseus Arthrobacter globiformis
Source*

DNA $\mathrm{G}+\mathrm{C}$ content (mol \%)

DSM 43067† (ATCC 19425, CCM 136, NCTC 5654)

$68 \cdot 2$

DSM 43380 (M. Goodfellow strain A32)

$68 \cdot 2$

$67 \cdot 7$

66.0

DSM 43121 (IMRU 754)

DSM 43122 (IMRU 780)

DSM 43123 (IMRU 1260)

DSM 43383

DSM 43382 (C. Philpot strain 1065)

DSM 43384 (M. Goodfellow strain A35)

DSM 43117 (IMRU 513)

DSM 43118 (IMRU 755)

DSM 43357† (ATCC 27296)

DSM 43144† (ATCC 27297)

DSM 43358+ (ATCC 27299)

DSM 43550

DSM 43459† (ATCC 27298)

DSM 43461 $\dagger$ (ATCC 27887)

DSM 43462 $\uparrow$ (ATCC 27888)

DSM $43551 \dagger$

DSM 43552†

DSM 43553†

DSM 43556

DSM $43555 \dagger$

ATCC $31588+$

DSM 43111+(ATCC 23218, NCTC 10488)

DSM 43235 (ATCC 23219, NCTC 10489)

DSM 43377 (M. Goodfellow, strain A92)

DSM 43378 (IMRU 714)

DSM 43021 † (ATCC 12428)

DSM 40236† (ATCC 23345, ISP 5236)

DSM 20124† (ATCC 8010, NCTC 8907)
$68 \cdot 0$

$68 \cdot 0$

$66 \cdot 0$

$67 \cdot 3$

$65 \cdot 5$

$67 \cdot 2$

$65 \cdot 5$

$68 \cdot 3$

68.5

69.0

$69 \cdot 0$

66.4

67.6

$68 \cdot 1$

$66 \cdot 2$

$66 \cdot 2$

$68 \cdot 1$

$66 \cdot 2$

$68 \cdot 1$

69.7

69.0

68.5

64.0

$64 \cdot 5$

$70 \cdot 3$

71.0

63.4

* Abbreviations: ATCC, American Type Culture Collection, Rockville, Md., U.S.A.; CCM, Czechoslovak Collection of Micro-organisms, Brno, Czechoslovakia; DSM, Deutsche Sammlung von Mikroorganismen, Gottingen, F.R.G.; M. Goodfellow, Department of Microbiology, Newcastle upon Tyne, U.K.; IMRU, Institute of Microbiology, Rutgers, The State University, New Brunswick, N.J., U.S.A.; ISP, International Streptomyces Project; NCTC, National Collection of Type Cultures, London, U.K.; C. Philpot, London School of Hygiene and Tropical Medicine, London, U.K.

† Type strain.

pelletieri DSM 43382, which are specifically related (75\%), homology values found between strains of $A$. madurae, A. pelletieri, $\boldsymbol{A}$. verrucosospora, and $A$. citrea DSM 43061 range between 25 and $44 \%$. Actinomadura malachitica DSM 43462, and ' $A$. kijaniata' ATCC 31588 show homology values of only 18 to $25 \%$ to the reference DNAs, while representatives of the second Actinomadura rRNA-cluster, as well as strains of Nocardiopsis and Streptosporangium show even lower homology values (7-12\%). Those Actinomadura strains which did not group with $A$. madurae DSM 43067 in the DNA-rRNA cistron similarity study do not form a genetically coherent group. Using reference [ $\left.{ }^{3} \mathrm{H}\right] \mathrm{DNA}$ of $A$. pusilla DSM 43357 and $A$. roseoviolacea DSM 43144 and $\left[{ }^{35}\right.$ S]DNA of $A$. libanotica DSM 43554, these three species, together with $A$. roseola DSM 43551 and DSM 43552 and $A$. ferruginea DSM 43553 form a distinct cluster in which DNA homology values are as high (Table 4) as those found among members of the $A$. madurae rRNA-cluster (Table 3). Four strains of Actinomadura, namely A. spadix DSM 43459, $A$. spiralis DSM 43555, $A$. madurae DSM 43121 and $A$. madurae DSM 43123, show little DNA homology to members of the two clusters formed around $A$. madurae and $A$. pusilla, or to each other (Table 4). 
Table 2. Melting points $\left(\mathrm{T}_{m(e)}\right)$ of DNA-23S rRNA duplexes of ${ }^{3} \mathrm{H}$-labelled $23 \mathrm{~S}$ RNA from Actinomadura madurae DSM 43067, and DNA from representatives of Actinomadura, Nocardiopsis Streptosporangium, Streptomyces and Arthrobacter

\begin{tabular}{|c|c|c|}
\hline \multicolumn{2}{|c|}{ Filter-bound DNA from strain: } & $T_{m(e)}$ \\
\hline Actinomadura madurae & DSM 43067 & $83 \cdot 7$ \\
\hline Actinomadura madurae & DSM 43380 & $84 \cdot 0$ \\
\hline Actinomadura madurae & DSM 43381 & $80 \cdot 8$ \\
\hline Actinomadura madurae & DSM 43122 & $81 \cdot 1$ \\
\hline Actinomadura pelletieri & DSM 43382 & $79 \cdot 8$ \\
\hline Actinomadura pelletieri & DSM 43383 & $79 \cdot 1$ \\
\hline Actinomadura pelletieri & DSM 43118 & $79 \cdot 6$ \\
\hline Actinomadura verrucosospora & DSM 43358 & $81 \cdot 0$ \\
\hline Actinomadura malachitica & DSM 43462 & $81 \cdot 2$ \\
\hline 'Actinomadura kijaniata' & ATCC 31588 & $79 \cdot 0$ \\
\hline Actinomadura citrea & DSM 43461 & $79 \cdot 2$ \\
\hline Actinomadura ferruginea & DSM 43553 & $73 \cdot 9$ \\
\hline Actinomadura roseola & DSM 43551 & $74 \cdot 8$ \\
\hline Actinomadura pusilla & DSM 43357 & $74 \cdot 0$ \\
\hline Actinomadura roseoviolacea & DSM 43144 & $75 \cdot 2$ \\
\hline Actinomadura libanotica & DSM 43556 & $74 \cdot 4$ \\
\hline Actinomadura spiralis & DSM 43555 & $74 \cdot 4$ \\
\hline Actinomadura spadix & DSM 43459 & $75 \cdot 8$ \\
\hline Actinomadura madurae & DSM 43121 & $73 \cdot 1$ \\
\hline Actinomadura madurae & DSM 43123 & $73 \cdot 0$ \\
\hline Nocardiopsis dassonvillei & DSM 43111 & 73.7 \\
\hline Nocardiopsis dassonvillei & DSM 43235 & $71 \cdot 7$ \\
\hline Nocardiopsis dassonvillei & DSM 43377 & $73 \cdot 7$ \\
\hline Nocardiopsis dassonvillei & DSM 43378 & $72 \cdot 5$ \\
\hline Streptosporangium roseum & DSM 43021 & $73 \cdot 8$ \\
\hline Streptomyces griseus & DSM 40236 & $73 \cdot 2$ \\
\hline Arthrobacter globiformis & DSM 20124 & 63.9 \\
\hline
\end{tabular}

The homology values for four strains of Nocardiopsis dassonvillei, obtained with the DNAfilter method and the spectrophotometrical method, are shown in Tables 5 and 6. Strains of $N$. dassonvillei group into two pairs of highly related organisms, which are moderately related to each other (36-38\%). No relationship could be detected between strains of $N$. dassonvillei and representatives of Actinomadura.

\section{Biochemical characteristics}

We could reconfirm that meso-diaminopimelic acid is the diagnostic amino acid in cell walls of all Actinomadura and Nocardiopsis strains investigated (Lechevalier \& Lechevalier, 1970). Tables 7 and 8 show the menaquinone, whole cell sugar composition and the fatty acid composition of all organisms tested.

The analysis of the menaquinones proved difficult. Using Li-Chrosorb RP 18 as a stationary phase, menaquinones of $A$. madurae DSM 43067 were found to be of the MK-9(H4)-, MK9(H6)-, and MK-9(H8)-types, similar to those found in Streptomyces (Collins \& Jones, 1981; Batrakov \& Bergelson, 1978). However, using the silver-loaded ion exchanger as a stationary phase (Kroppenstedt, 1982b), the retention times were different. These differences in the separation behaviour of menaquinones of the same number of isoprenoid units and double bonds depends upon the position at which the saturation of double bonds occur. While menaquinones of the MK-9(H6)-type in Streptomyces olivaceus are saturated at positions II, III, and IX (Batrakov \& Bergelson, 1978), that of $A$. madurae is saturated at positions II, III, and VIII (Aoki et al., 1980; Yamada et al., 1982). Our analysis of the menaquinones of the MK9(H4)-, and MK-9(H8)-types in strains of Actinomadura and Nocardiopsis also show retention times different to those of the same types found in S. olivaceus (Batrakov \& Bergelson, 1978), indicating differences in the position of saturation in these isomers too. The exact position of 
Table 3. Percentage DNA homologies among strains of the Actinomadura madurae rRNAcluster and other Actinomadura strains

Filter-bound DNA from strain:

\begin{tabular}{|c|c|c|c|c|c|}
\hline & & & A fron & in: & \\
\hline & DSM & DSM & DSM & DSM & DSM \\
\hline & 43067 & 43122 & 43383 & 43384 & 43118 \\
\hline DSM 43067 & 100 & 44 & 29 & 28 & 30 \\
\hline DSM 43380 & 96 & 48 & 32 & 31 & 33 \\
\hline DSM 43122 & 46 & 100 & 42 & 44 & 45 \\
\hline DSM 43381 & 33 & 58 & 37 & 37 & 40 \\
\hline DSM 43383 & 26 & 32 & 100 & 96 & 100 \\
\hline DSM 43384 & 25 & 33 & 98 & 100 & 100 \\
\hline DSM 43118 & - & 32 & - & 98 & 100 \\
\hline DSM 43117 & 29 & 38 & 90 & 85 & 89 \\
\hline DSM 43382 & 39 & 75 & 31 & 31 & 33 \\
\hline DSM 43358 & 32 & 35 & - & 31 & - \\
\hline DSM 43550 & 35 & 39 & - & 30 & 32 \\
\hline DSM 43461 & 38 & 42 & - & 30 & 33 \\
\hline DSM 43462 & 20 & 22 & - & 18 & 20 \\
\hline ATCC 31588 & 20 & 25 & - & 18 & 19 \\
\hline DSM 43357 & 7 & 11 & 9 & 11 & 11 \\
\hline DSM 43144 & 7 & 7 & 8 & - & 9 \\
\hline DSM 43459 & 11 & 8 & 7 & - & 10 \\
\hline DSM 43551 & 8 & - & 8 & - & 9 \\
\hline DSM 43121 & 8 & 9 & 10 & 10 & 9 \\
\hline DSM 43123 & 11 & 12 & 11 & 11 & 12 \\
\hline DSM 43111 & 8 & - & 9 & 9 & 7 \\
\hline DSM 43235 & 7 & 10 & 9 & -- & 8 \\
\hline DSM 43021 & 8 & - & 7 & - & 8 \\
\hline
\end{tabular}

Actinomadura madurae Actinomadura madurae Actinomadura madurae Actinomadura madurae Actinomadura pelletieri Actinomadura pelletieri Actinomadura pelletieri Actinomadura pelletieri Actinomadura pelletieri Actinomadura verrucosospora Actinomadura verrucosospora Actinomadura citrea Actinomadura malachitica 'Actinomadura kijaniata' Actinomadura pusilla Actinomadura roseoviolacea Actinomadura spadix Actinomadura roseola Actinomadura madurae Actinomadura madurae Nocardiopsis dassonvillei Nocardiopsis dassonvillei Streptosporangium roseum

-, Not determined.

Table 4. Percentage DNA homologies among Actinomadura pusilla, Actinomadura roseoviolacea, Actinomadura libanotica, Actinomadura spadix and Actinomadura spiralis and other Actinomadura strains

Filter-bound DNA from strain:

$\overbrace{\begin{array}{l}\text { DSM } \\ 43357\end{array}}^{\begin{array}{r}\text { DSM } \\ 43144\end{array}}$

\begin{tabular}{|c|c|}
\hline SM & DSM \\
\hline 43556 & 43459 \\
\hline
\end{tabular}

Actinomadura pusilla
Actinomadura roseoviolacea
Actinomadura libanotica
Actinomadura roseola
Actinomadura roseola
Actinomadura ferruginea
Actinomadura spadix
Actinomadura spiralis
Actinomadura madurae
Actinomadura madurae
Actinomadura madurae
Actinomadura madurae
Actinomadura pelletieri
Nocardiopsis dassonvillei
Streptosporangium roseum

$\begin{array}{rr}100 & 27 \\ 31 & 100 \\ 25 & 31 \\ 29 & 33 \\ 29 & 29 \\ 26 & 26 \\ 11 & 9 \\ 10 & 12 \\ 7 & 7 \\ 10 & 10 \\ 7 & 7 \\ 9 & 11 \\ 9 & 8 \\ 7 & 6 \\ 13 & 13\end{array}$

22
26
85
24
25
24
9
8
7
7
7
8
8
10
15

$\begin{array}{rr}7 & 7 \\ 9 & 10 \\ 9 & 11 \\ 12 & 12 \\ 12 & 14 \\ 10 & 11 \\ 100 & 10 \\ 10 & 100 \\ 8 & 8 \\ 5 & 5 \\ 12 & 11 \\ 13 & 13 \\ 13 & 12 \\ 9 & 10 \\ 9 & 8\end{array}$


Table 5. Percentage DNA homologies among strains of Nocardiopsis dassonvillei DSM 43235 and DSM 43377 and various Actinomadura strains

Filter-bound DNA from strain:

$\overbrace{\begin{array}{l}\text { DSM } \\ 43235\end{array}}^{\left[{ }^{35} \text { S]DNA from strain: }\right.}$

Nocardiopsis dassonvillei
Nocardiopsis dassonvillei
Nocardiopsis dassonvillei
Nocardiopsis dassonvillei
Actinomadura madurae
Actinomadura madurae
Actinomadura pelletieri
Actinomadura verrucosospora
Actinomadura verrucosospora
Actinomadura citrea
Actinomadura malachitica
Actinomadura kijaniata'
Actinomadura pusilla
Actinomadura roseoviolacea
Actinomadura roseola
Actinomadura roseola
Actinomadura ferruginea
Actinomadura libanotica
Actinomadura spiralis
Actinomadura spadix

DSM 4311

DSM 43235

DSM 43377

DSM 43378

DSM 43067

DSM 43381

DSM 43384

DSM 43358

DSM 43550

DSM 43461

DSM 43462

ATCC 31588

DSM 43357

DSM 43144

DSM 43551

DSM 43552

DSM 43553

DSM 43556

DSM 43555

DSM 43459

$\begin{array}{rr}100 & 38 \\ 100 & 38 \\ 38 & 100 \\ 36 & 98 \\ 8 & 11 \\ 9 & - \\ 9 & - \\ 11 & - \\ 11 & - \\ 12 & - \\ 11 & - \\ 12 & - \\ 7 & - \\ 10 & - \\ 12 & - \\ 12 & - \\ 11 & - \\ 9 & - \\ 11 & - \\ 10 & 11\end{array}$

-, Not determined.

Table 6. Percentage DNA homologies among various strains of Nocardiopsis dassonvillei and Actinomadura using the spectrophotometric reassociation method and comparison of homologies with those obtained by the DNA-filter method

\begin{tabular}{|c|c|c|}
\hline \multirow[b]{2}{*}{ DNA from strain: } & \multicolumn{2}{|c|}{ DNA homology $(\%)$} \\
\hline & $\begin{array}{l}\text { Spectrophotometric } \\
\text { method }\end{array}$ & $\begin{array}{l}\text { DNA-filter } \\
\text { method }\end{array}$ \\
\hline \multicolumn{3}{|l|}{ Nocardiopsis dassonvillei: } \\
\hline DSM $43235^{*} \times$ DSM 43111 & 99 & 100 \\
\hline DSM $43111 \times$ DSM 43378 & 35 & - \\
\hline DSM $43235^{*} \times$ DSM 43378 & 35 & 36 \\
\hline \multicolumn{3}{|l|}{ Actinomadura roseola: } \\
\hline DSM $43551 \times$ DSM 43552 & 100 & - \\
\hline \multicolumn{3}{|l|}{ Actinomadura roseola DSM $43551 \times$} \\
\hline Actinomadura ferruginea DSM 43553 & 26 & - \\
\hline \multicolumn{3}{|l|}{ Actinomadura roseoviolacea DSM $43144^{*} \times$} \\
\hline Actinomadura roseola DSM 43552 & 29 & 33 \\
\hline \multicolumn{3}{|l|}{ Actinomadura pusilla DSM $43357 \times$} \\
\hline Actinomadura roseoviolacea DSM 43144 & 40 & 3 \\
\hline \multicolumn{3}{|l|}{ Actinomadura verrucosospora: } \\
\hline DSM $43358 \times$ DSM 43550 & 96 & - \\
\hline
\end{tabular}


Table 7. Menaquinone isoprenologue and whole cell sugar composition

\begin{tabular}{|c|c|c|c|c|c|c|c|c|c|c|c|c|}
\hline \multirow[b]{3}{*}{ Strain } & & \multicolumn{11}{|c|}{ Menaquinone isoprenologue and degree of hydrogenation $\dagger$} \\
\hline & & \multicolumn{6}{|c|}{ MK-9 } & \multicolumn{5}{|c|}{ MK-10 } \\
\hline & & $\mathbf{H}_{0}$ & $\mathrm{H}_{2}$ & $\mathrm{H}_{4}{ }^{* \ddagger}$ & $\mathrm{H}_{6}{ }^{*} \S$ & $\mathrm{H}_{6} \| \mathrm{H}_{8} * q$ & $\mathbf{H}_{8}+\dagger$ & $\mathbf{H}_{0}$ & $\mathrm{H}_{2}$ & $\mathrm{H}_{4}$ & $\mathrm{H}_{6}$ & $\mathbf{H}_{8}$ \\
\hline 1. Actinomadura madurae & DSM 43067 & & & & 75 & 12 & 11 & & & & & \\
\hline 2. Actinomadura madurae & DSM 43380 & & & & 74 & 10 & 8 & & & & & \\
\hline 3. Actinomadura madurae & DSM 43381 & & & 8 & 54 & 11 & 10 & & & 11 & & \\
\hline 4. Actinomadura madurae & DSM 43122 & & & & 30 & 64 & & & & & & \\
\hline 5. Actinomadura pelletieri & DSM 43382 & & & 11 & 75 & 6 & 8 & & & & & \\
\hline 6. Actinomadura pelletieri & DSM 43384 & & & 14 & 80 & & & & & & & \\
\hline 7. Actinomadura pelletieri & DSM 43117 & & & 20 & 74 & & & & & & & \\
\hline 8. Actinomadura pelletieri & DSM 43118 & & & 20 & 44 & 21 & & & & & & \\
\hline 9. Actinomadura pelletieri & DSM 43383 & & & 7 & 65 & 9 & 16 & & & & & \\
\hline 10. Actinomadura verrucosospora & DSM 43358 & & & 11 & 85 & & & & & & & \\
\hline 11. Actinomadura verrucosospora & DSM 43550 & & & & 65 & 16 & 18 & & & & & \\
\hline 12. Actinomadura malachitica & DSM 43462 & & & 10 & 74 & 8 & 6 & & & & & \\
\hline 13. Actinomadura citrea & DSM 43461 & & & & 79 & 8 & & & & & & \\
\hline 14. Actinomadura pusilla & DSM 43357 & 15 & 20 & 60 & & & & & & & & \\
\hline 15. Actinomadura roseoviolacea & DSM 43144 & & 18 & 75 & & & & & & & & \\
\hline 16. Actinomadura libanotica & DSM 43556 & & 19 & 75 & & & & & & & & \\
\hline 17. Actinomadura roseola & DSM 43551 & & 26 & 68 & & & & & & & & \\
\hline 18. Actinomadura roseola & DSM 43552 & & 29 & 62 & & & & & & & & \\
\hline 19. Actinomadura ferruginea & DSM 43553 & 13 & 35 & 40 & & & & & & & & \\
\hline 20. Actinomadura spadix & DSM 43459 & & 6 & 50 & & 40 & & & & & & \\
\hline 21. Actinomadura spiralis & DSM 43555 & & 15 & 80 & & & & & & & & \\
\hline 22. Actinomadura madurae & DSM 43121 & & 10 & 82 & 8 & & & & & & & \\
\hline 23. Actinomadura madurae & DSM 43123 & & & & ND & & & & & & & \\
\hline 24. Nocardiopsis dassonvillei & DSM 43111 & & & 9 & & & & 6 & 7 & 39 & 27 & \\
\hline 25. Nocardiopsis dassonvillei & DSM 43235 & & & & & & & & 20 & 27 & 28 & 10 \\
\hline 26. Nocardiopsis dassonvillei & DSM 43377 & & & 32 & 18 & & & & & 18 & 15 & \\
\hline 27. Nocardiopsis dassonvillei & DSM 43378 & & & 33 & 17 & & & & & 24 & 20 & \\
\hline
\end{tabular}

ND, Not determined.

$(+)$, Trace only.

* Menaquinones found to be different from those of streptomycetes.

$\dagger$ Only those menaquinones are shown that are present in more than $5 \%$ of total menaquinones.

$\ddagger$ Menaquinones show other retention times on both columns as MK- $9\left(\mathrm{H}_{4}\right)$ of Streptomyces olivaceus (Batrakov \& Bergelson, 1978).

$\S$ Menaquinones show the same retention times on both columns as MK-9(II,III,VIII-H $)_{6}$ of Actinomadura madurae (Yamada et al., 1982).

$\|$ Menaquinones show the same retention times on both columns as MK-9(II,III,IX-H $\left.{ }_{6}\right)$ of Streptomyces olivaceus (Batrakov \& Bergelson, 1978).

I Menaquinones show other retention times on both columns as MK-9(II,III,VII,IX-H ${ }_{8}$ ) of Streptomyces olivaceus (Batrakov \& Bergelson, 1978).

if Menaquinones show the same retention times on both columns as MK-9(II,III,VII,IX-H $\left.{ }_{8}\right)$ of Streptomyces olivaceus (Batrakov \& Bergelson, 1978).

saturation, however, can only be detected by mass spectroscopy, which has not been done in this study. The menaquinones found to be different from those of streptomycetes are marked with an asterisk in the text and in Table 7.

Members of the $A$. madurae rRNA-cluster show the following characteristics: menaquinones are predominantly of the MK-9(H6)*-type, as characterized by Aoki et al. (1980) and Yamada et al. (1982). In some strains additional menaquinones of the MK-9(H4)*-, MK-9(H8)*- and MK9(H8)-types occur. Ribose, mannose, glucose, galactose and madurose are almost always present as whole cell sugars, though in some strains the latter sugar is present in very small amounts only. Arabinose and galactose are always missing. The fatty acid composition of all members of this cluster is uniform. The percentage of hexadecanoic acid $(16: 0)$ is high $(11-50 \%)$, exceeding in most strains that of 14-methylpentadecanoic acid (i-16:0). The exceptions are A. pelletieri strains DSiM 43118, DSM 43383 and DSM 43384 and $A$. citrea DSM 43461. Iso- and anteiso- 
Table 7.-continued

\begin{tabular}{|c|c|c|c|c|c|c|c|}
\hline & & & & cell wall su & & & \\
\hline & Madurose & Xylose & Arabinose & Galactose & Glucose & Mannose & Ribose \\
\hline 1. & + & - & - & - & + & - & + \\
\hline 2. & + & + & + & + & + & + & + \\
\hline 3. & $(+)$ & - & - & + & ++ & $(+)$ & + \\
\hline 4. & + & - & - & - & ++ & - & + \\
\hline 5. & + & - & + & $(+)$ & + & - & + \\
\hline 6. & $(+)$ & - & - & $(+)$ & ++ & $(+)$ & + \\
\hline 7. & + & - & - & + & + & + & + \\
\hline 8. & & & & ND & & & \\
\hline 9. & + & _ & - & $(+)$ & ++ & $(+)$ & + \\
\hline 10. & $(+)$ & - & - & $(+)$ & ++ & $(+)$ & + \\
\hline 11. & & & & ND & & & \\
\hline 12. & + & - & - & + & ++ & $(+)$ & + \\
\hline 13. & $(+)$ & - & - & + & ++ & + & + \\
\hline 14. & $(+)$ & - & - & $(t)$ & ++ & + & + \\
\hline 15. & $(+)$ & - & - & $(+)$ & ++ & + & + \\
\hline 16. & $(+)$ & - & - & + & ++ & $(+)$ & + \\
\hline 17. & + & - & - & ++ & + & - & - \\
\hline 18. & $(+)$ & - & - & + & + & - & + \\
\hline 19. & $(+)$ & - & - & + & ++ & - & + \\
\hline 20. & + & - & - & - & + & + & $(+)$ \\
\hline 21. & + & + & - & - & + & + & + \\
\hline 22. & + & - & - & ++ & ++ & - & + \\
\hline 23. & + & + & + & + & ++ & - & + \\
\hline 24. & - & - & - & - & + & - & + \\
\hline 25. & - & - & - & $(+)$ & + & - & - \\
\hline 26. & - & - & _- & - & + & + & + \\
\hline 27. & - & - & - & - & + & + & + \\
\hline
\end{tabular}

branched fatty acids with an odd number of carbon atoms (iso/anteiso, 15/17) are almost missing. Among the 10-methyl-branched fatty acids, 10-methyloctadecanoic acid (18-me, tuberculostearic acid) is predominant over 10-methylheptadecanoic acid, which occurs mostly in less than $10 \%$ of total fatty acids.

All members of the A. pusilla-cluster have a similar menaquinone composition with the MK9(H4)*-type dominating. Menaquinone of the MK-9(H2)-type is present in all organisms. Ribose, glucose, galactose and madurose are present, though the latter sugar mostly in trace amounts. Mannose is missing in strains of $\boldsymbol{A}$. roseola and $\boldsymbol{A}$. ferruginea, while xylose and arabinose are missing in all strains. The most predominant feature of the other strains of this cluster is the high amount of 10-methylheptadecanoic acid (17-me) (17-33\%). Hexadecanoic acid (16:0) (1-15\%) is less common than in the A. madurae-rR NA cluster. Actinomadura spadix, $A$. spiralis, and the two $A$. madurae strains DSM 43121 and DSM 43123 are chemotaxonomically diverse and none of them show a specific similarity to members of the A. madurae-and A.pusillaclusters.

Strains of Nocardiopsis synthesize menaquinones with a long isoprenylic side chain [MK $\left.10\left(\mathrm{H}_{2}, \mathrm{H}_{4}, \mathrm{H}_{6}\right)\right]$. Strains DSM 43377 and DSM 43378 in addition have remarkably high amounts of menaquinones of the $\mathrm{MK} 9\left(\mathrm{H}_{4}{ }^{*}, \mathrm{H}_{6}{ }^{*}\right)$-types. Madurose is not present in whole cell hydrolysates, whereas ribose is present in all strains. The other sugars are not uniformly distributed. All strains have the same fatty acid composition, resembling in some aspects that of members of the $A$. pusilla-cluster. Hexadecanoic acid is present in small amounts only $(1-2 \%)$, while 14-methylpentadecanoic acid (15-40\%), 14-methylhexadecanoic acid (10-25\%), and 10methyloctadecanoic acid $(11-18 \%)$ are predominant. This fatty acid pattern can easily be distinguished from the two patterns found in members of the two Actinomadura-clusters by its high amount of terminally branched fatty acids with an odd number of carbon atoms in the straight chain residues [iso- and anteiso $\mathrm{C} 15$ and $\mathrm{C} 17$ fatty acids $(17-44 \%)$ ]. 
Table 8. Percentage fatty acid composition of the tested strains

Abbreviations for fatty acids are illustrated by the following examples: straight-chain saturated : hexadecanoic acid $(16: 0)$, monounsaturated :octadecanoic acid (oleic acid, $18: 1$ ), iso-branched:14methylheptadecanoic acid (iso-16), anteiso-branched : 14-methylhexadecanoic acid (anteiso-17), 10methyl-branched : 10-methyloctadecanoic acid (tuberculostearic acid, 18-me).

\section{Strain}

1. Actinomadura madurae

2. Actinomadura madurae

3. Actinomadura madurae

4. Actinomadura madurae

5. Actinomadura pelletieri

6. Actinomadura pelletieri

7. Actinomadura pelletieri

8. Actinomadura pelletieri

9. Actinomadura pelletieri

10. Actinomadura verrucosospora

11. Actinomadura verrucosospora

12. Actinomadura malachitica

13. Actinomadura citrea

14. Actinomadura pusilla

15. Actinomadura roseoviolacea

16. Actinomadura libanotica

17. Actinomadura roseola

18. Actinomadura roseola

19. Actinomadura ferruginea

20. Actinomadura spadix

21. Actinomadura spiralis

22. Actinomadura madurae

23. Actinomadura madurae

24. Nocardiopsis dassonvillei

25. Nocardiopsis dassonvillei

26. Nocardiopsis dassonvillei

27. Nocardiopsis dassonvillei

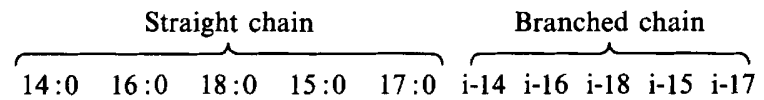

DSM 43067
DSM 43380
DSM 43381
DSM 43122
DSM 43382
DSM 43384
DSM 43117
DSM 43118
DSM 43383
DSM 43358
DSM 43550
DSM 43462
DSM 43461
DSM 43357
DSM 43144
DSM 43556
DSM 43551
DSM 43552
DSM 43553
DSM 43459
DSM 43555
DSM 43121
DSM 43123
DSM 43111
DSM 43235
DSM 43377
DSM 43378

$14: 0 \quad 16: 0 \quad 18: 0 \quad 15: 0$

$17: 0$

\begin{tabular}{|c|c|}
\hline 1 & 30 \\
\hline 2 & 38 \\
\hline \multirow[t]{2}{*}{3} & 39 \\
\hline & 17 \\
\hline \multirow[t]{5}{*}{2} & 28 \\
\hline & 31 \\
\hline & 33 \\
\hline & 24 \\
\hline & 14 \\
\hline 4 & 50 \\
\hline 2 & 39 \\
\hline 2 & 34 \\
\hline 1 & 11 \\
\hline 2 & 15 \\
\hline & 5 \\
\hline 2 & 4 \\
\hline 2 & 2 \\
\hline 2 & 4 \\
\hline 1 & 1 \\
\hline & 2 \\
\hline 1 & 17 \\
\hline 1 & 38 \\
\hline & 12 \\
\hline 1 & 1 \\
\hline & 2 \\
\hline & $\begin{array}{l}1 \\
1\end{array}$ \\
\hline
\end{tabular}

$\begin{array}{r}4 \\ 7 \\ 7 \\ 1 \\ 3 \\ 5 \\ 1 \\ 1 \\ 5 \\ 3 \\ 4 \\ 4 \\ 1 \\ 1 \\ 1 \\ 1 \\ 1 \\ 7 \\ 8 \\ 5 \\ 7 \\ 19 \\ 6 \\ 3 \\ \hline\end{array}$

$\begin{array}{rr}1 \\ 1 \\ 1 \\ 6 & \\ 7 & \\ & \\ 3 & 1 \\ 2 & \\ 1 & \\ & \\ 1 & \\ 1 & \\ 2 & \\ 11 & 13 \\ 6 & \\ 5 & \\ 5 & \\ 11 & 1 \\ 3 & \\ 1 & \\ 2 & \\ 1 & \\ 2 & \\ & \\ 1 & \end{array}$

$\begin{array}{rrrrr} & 4 & & & \\ & 7 & 1 & & \\ & 7 & & & \\ & 14 & 2 & & \\ & 5 & & & \\ & 35 & 6 & & \\ & 16 & 2 & & \\ & 45 & 4 & & \\ & 60 & 3 & & \\ & 3 & & & \\ & 5 & & & \\ & 8 & & & \\ 1 & 40 & 2 & & \\ 3 & 20 & & 5 & 1 \\ 3 & 41 & & 4 & \\ 2 & 27 & & 4 & 1 \\ 5 & 26 & & 5 & 1 \\ 3 & 21 & & 6 & \\ 1 & 36 & 3 & & \\ 1 & 50 & 2 & & 2 \\ & 7 & & 6 & 1 \\ & 5 & 1 & & \\ & 27 & 9 & & \\ 1 & 15 & 3 & 9 & 4 \\ 7 & 26 & 4 & & \\ 32 & 6 & & 2 & 4 \\ 2 & 40 & 10 & & 3\end{array}$

\section{DISCUSSION}

The DNA G + C content of Actinomadura strains is similar to that reported by Bradley et al. (1973) and intermediate between the values of $74 \mathrm{~mol} \%$ reported by Lechevalier et al. (1971) and $65 \mathrm{~mol} \%$ reported by Mordarski et al. (1977) and Bradley \& Mordarski (1976) for A. madurae. The $\mathrm{G}+\mathrm{C}$ values of Nocardiopsis dassonvillei show a broader range, separating the strains into two groups.

Our data on the fatty acid composition of Actinomadura and Nocardiopsis strains is only partially in accord with those published by Agre et al. (1975), although A. madurae and A. pusilla were also clearly separated from strains of Nocardiopsis, $A$. pusilla and $A$. roseoviolacea in the latter study. Qualitative and quantitative differences in the fatty acid composition are obvious. To give some examples, palmitoleic acid, which in our study is present only in small amounts in strains of $A$. madurae and $A$. pusilla $(0-6 \%)$, is a dominant fatty acid (13-56\%) in strains of these two species in the study of Agre et al. (1975). While we found high amounts of 10-methyl branched fatty acids in strains of $N$. dassonvillei, $A$. pusilla and $A$. roseoviolacea, this component was not listed among the fatty acids in these strains by Agre et al. (1975); oleic acid, on the other hand, was reported to be present in $A$. pusilla and $A$. roseoviolacea, while we were unable to detect this component in these strains. Some of the contradicting results of the two studies may be explained by differences in the age of the organisms investigated, which may influence their fatty acid composition (Kroppenstedt, 1977). It is now established that a good resolution of isoand anteiso-branched fatty acids require at least a $6 \mathrm{~m}$ polar column (Kaneda, 1977) and that the very complicated mixtures of fatty acids occurring in some genera of the Actinomycetales (Ballio 
Table 8.--continued

\begin{tabular}{|c|c|c|c|c|c|c|c|c|c|c|c|c|}
\hline & $\begin{array}{r}\text { Bra } \\
\text { cl }\end{array}$ & & & & Unsatur & ted fatt & acids & & & & $\begin{array}{l}\text { Met } \\
\text { chai } \\
\text { chai }\end{array}$ & \\
\hline & ai -15 & ai -17 & $\mathrm{i}-16: 1$ & $\mathrm{i}-17: 1$ & $\mathrm{i}-18: 1$ & $15: 1$ & $16: 1$ & $17: 1$ & $18: 1$ & 16 & 17 & 18 \\
\hline 1. & & & & 2 & & & 2 & & 24 & 3 & 3 & 23 \\
\hline 2. & & & & & & & 2 & 1 & 8 & & 2 & 28 \\
\hline 3. & & & & & & & & & 12 & 1 & & 28 \\
\hline 4. & & & & & 1 & & 6 & 9 & 6 & & 14 & 17 \\
\hline 5. & & & & & & & 2 & 6 & 14 & & 4 & 13 \\
\hline 6. & & & & & & & & & 12 & & 2 & 6 \\
\hline 7. & & & & & & & 2 & 4 & 18 & & 2 & 8 \\
\hline 8. & & & & & 3 & & 2 & 2 & 8 & & & 6 \\
\hline 9. & & & & & 2 & & & 1 & 10 & 1 & 1 & 4 \\
\hline 10. & & & & & & & 2 & & 15 & & & 20 \\
\hline 11. & & & & & & & 4 & & 13 & 1 & & 31 \\
\hline 12. & & & & & & & 3 & & 3 & 3 & 2 & 39 \\
\hline 13. & & & & & 3 & & 2 & 2 & 5 & & 8 & 16 \\
\hline 14. & 1 & & & & & & & & & 7 & 18 & 3 \\
\hline 15. & & & & & & 5 & & & & 5 & 23 & 2 \\
\hline 16. & 20 & & 2 & & & & 2 & 8 & & 2 & 17 & 1 \\
\hline 17. & 3 & & 7 & & & & 2 & 2 & & 2 & 33 & 3 \\
\hline 18. & & & 2 & & & & & 10 & & & 30 & \\
\hline 19. & 2 & & 1 & & 9 & & & 14 & 3 & & 17 & 2 \\
\hline 20. & 2 & 15 & 10 & & 4 & & & 1 & 1 & 3 & 5 & \\
\hline 21. & 2 & 3 & 4 & & & & 2 & 6 & 34 & & 6 & \\
\hline 22. & & & & & & & 2 & 1 & 13 & 2 & 1 & 24 \\
\hline 23. & & & & & 8 & & & 3 & 9 & 2 & 3 & 16 \\
\hline 24. & 6 & 25 & & & 4 & & & 2 & 2 & & 3 & 15 \\
\hline 25. & 7 & 12 & & & & & & 2 & 6 & & & 11 \\
\hline 26. & 4 & 15 & & & 1 & & & 2 & 8 & & 3 & 18 \\
\hline 27. & 4 & 10 & & & 1 & & & 1 & 4 & 1 & 4 & 16 \\
\hline
\end{tabular}

\& Barcellona, 1968; Ballio et al., 1968) can only be resolved and identified by gas chromatography using capillary columns with different separation behaviours (Kroppenstedt, 1982a). In the light of these results it might be that Agre et al. (1977) misinterpreted 10methylheptadecanoic acid for oleic acid because both fatty acids show nearly the same retention time on a non-polar packed column.

The analysis of 13 species of Actinomadura by nucleic acid reassociation and rRNA cistron similarity studies points towards a phylogenetic heterogeneity of this genus. Using the $23 \mathrm{~S}$ rRNA of $A$. madurae DSM 43067 as a phylogenetic marker, six species of Actinomadura are clearly separated from the other Actinomadura strains investigated. Within the Actinomadura madurae rRNA-cluster, $A$. madurae, $A$. pelletieri, $A$. verrucosospora and $A$. citrea form a centre of moderately related species, as seen from the DNA homology values above $30 \%$, while ' $A$. kijaniata' and $A$. malachitica are more peripherally related. The specific relationship between $A$. madurae and $A$. pelletieri has been noticed in several earlier investigations. Strains of both species produce a prodigiosin-like pigment (Gerber \& Lechevalier, 1964, 1965) and they resembled each other in the composition of fatty acids (Agre et al., 1975), phospholipids (Lechevalier et al., 1977), polar lipids (Minnikin et al., 1977), and menaquinones (Yamada et al., 1977; Collins et al., 1977; Collins \& Jones, 1981). On the other hand, Goodfellow \& Pirouz (1982) in a numerical phenetic analysis found strains of $A$. madurae and $A$. pelletieri to cluster separately from each other, thus bringing into question the classification of these two species in one genus. In the same investigation $A$. citrea and $A$. verrucosospora clustered with $A$. madurae, which is consistent with our finding concerning the genetic and phenotypic relationship of these 
organisms. In contrast to our results, $A$. spadix, $A$. pusilla and A. roseoviolacea were also found to be members of the phenetically defined $A$. madurae-cluster. As mentioned by Goodfellow \& Pirouz (1982), the relationships determined by numerical phenetic analysis may be distorted by several factors involved in the tests and statistics used.

Actinomadura strains which do not belong to the $A$. madurae rRNA-cluster constitute a collection of moderately to slightly related organisms. Among these, A. pusilla, A. roseoviolacea, $A$. roseola, $A$. libanotica and $A$. ferruginea are geno- and phenotypically related. This has been shown before for $A$. pusilla and $A$. roseoviolacea by similarities in the fatty acid composition (Agre et al., 1975), which differs from that of $A$. madurae and relatives on the one hand, and from that of $N$. dassonvillei on the other. Actinomadura helvata, not included in these studies, is phenotypically very similar to members of the $A$. pusilla-cluster (R. M. Kroppenstedt, unpublished) suggesting that it is probably a member of this group.

Nacardiopsis, originally excluded from Actinomadura on the basis of morphological and biochemical grounds (Meyer, 1976), bears little DNA and rRNA similarity to members of Actinomadura. Despite a superficial resemblance in the peptidoglycan type and the DNA G + C content, the low degree of genetic relatedness of these two taxa is also reflected in differences in the composition of menaquinones, polar lipids, fatty acids, whole cell sugars and in spore-chain morphology. Our data indicate that the species $N$. dassonvillei contains two groups of remotely related strains, separated from each other not only by DNA homologies of about $30 \%$ but also in the menaquinone composition and the DNA G $+\mathrm{C}$ content.

We believe the genus Actinomadura should be restricted to members of the $A$. madurae rRNAcluster. The $\Delta T_{m(e)}$ values of the DNA-rRNA duplexes found among members of this cluster cover a narrow range of $5.4{ }^{\circ} \mathrm{C}$. Similar values have been found among Streptomyces, Streptosporangium and relatives, and Actinoplanes and relatives (Stackebrandt et al., 1981), Microbacterium and Curtobacterium (Döpfer et al., 1982), Staphylococcus (Kilpper-Bälz \& Schleifer, 1981) and various Gram-negative genera (De Smedt \& De Ley, 1977; Gillis \& De Ley, 1980). All other Actinomadura species show $\Delta T_{m(e)}$ values which are on average $6.5^{\circ} \mathrm{C}$ lower than the average $\Delta T_{m(e)}$ values of members of the $A$. madurae rRNA-cluster. These values are in the same order of magnitude as those found to separate members of different genera, e.g. Streptomyces from Streptosporangium and Actinoplanes (Stackebrandt et al., 1981), Curtobacterium and Microbacterium from Cellulomonas (Döpfer et al., 1982), Staphylococcus from Bacillus (Kilpper-Bälz \& Schleifer, 1981) and Actinomadura ( $A$. madurae rRNA-cluster) from Streptomyces (this study).

The taxonomic rank of the remaining Actinomadura species is not as clearcut. Whether or not the genotypically and phenotypically coherent cluster of $A$. pusilla, A. roseoviolacea, A. roseola, $A$. libanotica and $A$. ferruginea together constitute an individual genus cannot be deduced from the results presented here. However, the analysis of oligonucleotides of the 16S rRNA reveal that this group of organisms and Nocardiopsis are not phylogenetically closely related either to each other or to any genus of Actinomycetales so far investigated by this method (unpublished results).

Part of this work was supported by the Deutsche Forschungsgemeinschaft. The authors wish to thank Heike Pohla and Ralph Rosenstein for their valuable assistance in the nucleic acid hybridization work.

\section{REFERENCES}

Agre, N. S., Efimova, T. P. \& Guzeva, L. N. (1975). Heterogeneity of the genus Actinomadura Lechevalier and Lechevalier. Mikrobiologiya 44, 252-257.

AokI, K., Yamada, Y. \& TahaRA, Y. (1980). A new natural quinone, 2-methyl-3-II,III,VIII-hexahydromultiprenyl'-1,4-naphthoquinone. Agricultural and Biological Chemistry 44, 1993-1965.

Ballio, A. \& Barcellona, S. (1968). Relations chimiques et immunologiques chez les Actino- mycetales. I. Les acides gras de 43 souches d'Actinomycetales aerobies. Annales de l'Institut Pasteur 114, 121-137.

Ballio, A., Barcellona, S. \& Salvatori, T. (1968). Identification of 9,10-methylenehexadecanoic acid in some aerobic 'Actinomycetales' by a combined. gas chromatographic-mass spectroscopic technique. Journal of Chromatography 35, 211-212.

Batrakov, S. G. \& BeRgelson, L. D. (1978). Lipids of 
the streptomycetes. Structural investigation and biological interrelation. Chemistry and Physics of Lipids 21, 1-29.

Becker, B., Lechevalier, M. P. \& Lechevalier, H. A. (1965). Chemical composition of cell wall preparations from strains of various form genera of aerobic actinomycetes. Applied Microbiology 13, 236-243.

Bradley, S. G. \& Mordarski, M. (1976). Association of polydeoxyribonucleotides of deoxyribonucleic acids from nocardioform bacteria. In The Biology of Nocardiae, pp. 310-336. Edited by M. Goodfellow, G. H. Brownell \& J. A. Serrano. London: Academic Press.

Bradley, S. G., Brownell, G. H. \& Clark, J. (1973). Genetic homologies among Nocardiae and other actinomycetes. Canadian Journal of Microbiology 19 , 1007-1014.

Collins, M. D. \& Jones, D. (1981). Distribution of isoprenoid quinone structural types in bacteria and their taxonomic implication. Microbiological Reviews 45, 316-354.

Collins, M. D., Pirouz, T., Goodfellow, M. \& MinNikin, D. E. (1977). Distribution of menaquinones in actinomycetes and corynebacteria. Journal of General Microbiology 100, 221-230.

DE LEY, J. \& DE SMEDT, J. (1975). Improvements of the membrane filter method for DNA/rRNA hybridization. Antonie van Leeuwenhoek 41, 287-307.

De Ley, J., Cattoir, H. \& ReynaerTs, A . (1970). The quantitative measurement of DNA hybridization from renaturation rates. European Journal of Biochemistry 12, 133-142.

DENHARDT, D. T. (1966). A membrane-filter technique for the detection of complementary DNA. Biochemical and Biophysical Research Communications 23, 641-646.

DE SMEDT, J. \& DE LEY, J. (1977). Intra- and intergeneric similarities of Agrobacterium ribosomal ribonucleic acid cistrons. International Journal of Systematic Bacteriology 27, 222-240.

Döpfer, H., Stackebrandt, E. \& Fiedler, F. (1982). Nucleic acid hybridization studies on Microbacterium, Curtobacterium, Agromyces and related taxa. Journal of General Microbiology 128, 1697-1708.

Dunphy, P., Phillips, P. G. \& Brodie, A. F. (1971). Separation and identification of menaquinones from microorganisms. Journal of Lipid Research 12, 442449.

Frank-KAMENETSKII, M. D. (1971). Simplification of the empirical relationship between the melting temperature of DNA, its GC content and concentration of sodium ions in solution. Biopolymers 10, 26252628.

Gerber, N. N. \& Lechevalier, M. P. (1964). Phenazines and phenoxazinones from Waksmania aerata sp. nov. and Pseudomonas iodina. Biochemistry 3. 598-602.

Gerber, N. N. \& Lechevalier, M. P. (1965). 1,6Phenazinediol-5-oxide from micro-organisms. Biochemistry 4, 176-180.

GiLLIS, M. \& DE LEY, J. (1980). Intra- and intergeneric similarities of the ribosomal ribonucleic acid cistrons of Acetobacter and Gluconobacter. International Journal of Systematic Bacteriology 30, 7-27.
Gillis, M., De Ley, J. \& De Cleene, M. (1970). The determination of molecular weight of bacterial genome RNA from renaturation rates. European Journal of Biochemistry 12, 143-153.

Goodfellow, M. \& Pirouz, T. (1982). Numerical classification of sporoactinomycetes containing meso-diaminopimelic acid in the cell wall. Journal of General Microbiology 128, 503-527.

Horan, A. C. \& Brodsky, B. C. (1982). A novel antibiotic-producing Actinomadura, Actinomadura kijaniata sp. nov. International Journal of Systematic Bacteriology 32, 195-200.

KANEDA, T. (1977). Fatty acids of the genus Bacillus: an example of branched-chain preference. Bacteriological Reviews 41, 391-418.

KILPPER-B⿵̈LZ, R. \& SCHLEIFER, K. H. (1981). DNArRNA hybridization among staphylococci and some other Gram-positive bacteria. FEMS Microbiology Letters 10, 357-362.

KROPPENSTEDT, R. M. (1977). Untersuchungen zur Chemotaxonomie der Ordnung Actinomycetales Buchanan 1917. Ph.D thesis, University of Darmstadt, F.R.G.

KROPPENSTEDT, R. M. (1982a). Anwendung von Dünnschicht- und Gaschromatographie in der Bakterientaxonomie. GIT-Fachzeitschrift, Labor. Supplement 'Chromatographie', pp. 34-40.

KROPPENSTEDT, R. M. (1982b). Separation of bacterial menaquinones by HPLC using reverse phase (RP 18) and a silver loaded ion exchanger as stationary phases. Journal of Liquid Chromatography 5, 2359-2367.

KROPPENSTEDT, R M. \& KUTZNER, H. J. (1978), Biochemical taxonomy of some problem actinomycetes. Zentralblatt für Bakteriologie, Parasitenkunde, Infektionskrankhetten und Hygiene (I. Abteilung) Supplement 6, 125-133.

KROPPENSTEDT, R. M., KORN-WENDISCH, F., FOWLER, V. J. \& StackebrandT, E. (1981). Biochemical and molecular genetic evidence for a transfer of Actinoplanes armeniacus into the family Streptomycetaceae. Zentralblatt für Bakteriologie und Hygiene (I. Abteilung, Originale C) 2, 254-262.

Lechevalier, H. A., Lechevalier, M. P. \& Gerber, N. N. (1971). Chemical composition as a criterion in the classification of actinomycetes. Adrances in Applied Microbiology 14, 47-72.

Lechevalier, M. P. \& Gerber, N. N. (1970). The identity of madurose with 3-O-methyl-D-galactose Carbohydrate Research 13, 451-454.

Lechevalier, M. P. \& Lechevalier, H. (1970). Chemical composition as a criterion in the classification of aerobic actinomycetes. International Journal of Systematic Bacteriology 20, 435-444.

Lechevalier, M. P., De Bievre, C. \& Lechevalier, H. A. (1977). Chemotaxonomy of aerobic actinomycetes: phospholipid composition. Biochemical Sys. tematics and Ecology 5, 249-260.

Mandel, M. \& Marmur, J. (1968). Use of ultraviolet absorbance temperature profile for determining the guanine plus cytosine content of DNA. Methods in Enzymology 12B, 195-206.

MARMUR, J. (1961). A procedure for the isolation of deoxyribonucleic acid from microorganisms. Journal of Molecular Biology 3, 208-218. 
McConaughy, B. L., LaIRD, C. D. \& McCarthy, B. J. M. (1969). Nucleic acid reassociation in formamide. Biochemistry 8, 3289-3295.

MEYER, J. (1976). Nocardiopsis, a new genus of the order Actinomycetales. International Journal of Systematic Bacteriology 26, 487-493.

MeYer, S. A. \& SCHLEIFER, K. H. (1978). Deoxyribonucleic acid reassociation in the classification of coagulase-positive staphylococci. Archives of Microbiology 117, 183-188.

Minnikin, D. E., Alshamaony, L. \& Goodfellow, M. (1975). Differentiation of Mycobacterium, Nocardia and related taxa by thin-layer chromatographic analysis of whole-organism methanolysates. Journal of General Microbiology 88, 200-204.

Minnikin, D. E., Pirouz, T. \& Goodfellow, M (1977). Polar lipid composition in the classification of some Actinomadura species. International Journal of Systematic Bacteriology 27, 118-121.

Mordarski, M., Goodfellow, M., Szyba, K., PULVERER, G. \& TKACZ, A. (1977). Classification of the 'rhodochrous' complex and allied taxa based upon deoxyribonucleic acid reassociation. International Journal of Systematic Bacteriology 27, 31-37.

Skerman, V. B. D., McGowan, V. \& SNEath, P. H. A. (1980). Approved Lists of Bacterial Names. International Journal of Systematic Bacteriology 30, 225-420.

STACKEBRANDT, E. \& KANDLER, O. (1979). Taxonomy of the genus Cellulomonas, based on phenotypic characters and deoxyribonucleic acid-deoxyribonucleic acid homology and proposal of seven neotype strains. International Journal of Systematic Bacteriology 29, 273-282.

StACKebRaNDT, E. \& SCHLEIFER, K. H. (1983). Molecular systematics of actinomycetes and related organisms. In Actinomycete Biology. Edited by L. F. Bojalil \& L. Ortiz-Ortiz. New York: Academic Press (in the Press).
Stackebrandt, E. \& Woese, C. R. (1981 $a$ ). Towards a phylogeny of actinomycetes and related organisms. Current Microbiology 5, 131-136.

StackebrandT, E. \& Woese, C. R. $(1981 b)$. The evolution of prokaryotes. In Molecular and Cellular Aspects of Microbial Evolution, pp. 1-31. Edited by M. J. Carlile, J. F. Collins \& B. E. B. Moseley. Cambridge: Cambridge University Press.

STACKebrandT, E., WUNNER-FüsSL, B., Fowler, V. J. \& SCHLEIFER, K. H. (1981). Deoxyribonucleic acid homologies and ribosomal ribonucleic acid similarities among sporeforming members of the order Actinomycetales. International Journal of Systematic Bacteriology 31, 420-431.

STACKEBRANDT, E., KROPPENSTEDT, R. M. \& FowLER, V. J. (1983). A phylogenetic analysis of the family Dermatophilaceae. Journal of General Microbiology 129, 1831-1838.

Staneck, J. L. \& RoberTs, G. D. (1974). Simplified approach to identification of aerobic actinomycetes by thin-layer chromatography. Journal of Applied Microbiology 20, 266-272.

Williams, S. T. \& Wellington, E. M. H. (1981). The genera Actinomadura, Actinopolyspora, Excellospora, Microbispora, Microtetraspora, Nocardiopsis, Saccharopolyspora and Pseudonocardia. In The Prokaryotes, pp. 2103-2117. Edited by M. P. Starr, H. Stolp, H. G. Trüper, A. Balows \& H. G. Schlegel. Berlin, Heidelberg, New York: Springer.

Yamada, Y., Yamashita, M., TahaRA, Y. \& Kondo, K. (1977). The menaquinone system in the classification of the genus Actinomadura. Journal of Applied Microbiology 23, 311-335.

Yamada, Y., AokI, K. \& Tahara, Y. (1982). The structure of hexa-hydrogenated isoprenoid sidechain menaquinone with nine isoprene units isolated from Actinomadura madurae. Journal of General and Applied Microbiology 28, 321-329. 